\title{
ESTADO DA ARTE EM MECANISMOS DE RASTREAMENTO SOLAR
}

\author{
G. O. PRADO ${ }^{1,2}$, T. F. ULHOA ${ }^{2}$, J.J.R. DAMASCENO ${ }^{1}$, L.G.M. VIEIRA ${ }^{1}$ \\ ${ }^{1}$ Universidade Federal de Uberlândia, Departamento de Engenharia Química \\ ${ }^{2}$ Universidade Federal do Triângulo Mineiro, Departamento de Engenharia de Produção \\ E-mail para contato: webgop@gmail.com
}

\begin{abstract}
RESUMO - A energia solar é uma das fontes energéticas mais cobiçadas quando o assunto é desenvolvimento sustentável, porém, ainda carece de soluções para um melhor aproveitamento de captação e conversão. Uma das formas que otimizam essa captação é o rastreamento solar ou heliotropismo que é o nome dado ao fenômeno de orientação através do curso do Sol e pode ser utilizado tanto por equipamentos mecânicos, elétricos, como também por plantas, o que é o caso do girassol. Esse sistema rastreador pode ser empregado em concentradores solares que, essencialmente, dependem desse mecanismo para viabilidade funcional e, em aquecedores solares planos, que tradicionalmente são fixos. A movimentação pode ser feita utilizando apenas um eixo, no sentido da altitude ou do azimute, ou ainda utilizando dois eixos, que variam nos dois sentidos. Este trabalho tem como intuito facilitar o estudo de tecnologias de rastreamento solar de um e de dois eixos, utilizando-se, para isso, da análise de referências da área.
\end{abstract}

\section{INTRODUÇÃO}

O uso da energia solar é diversificado e atende desde o aquecimento de tubulações até a produção de energia elétrica. Possui também como característica essencial, o fato de ser renovável e de ser praticamente inesgotável, atributos esses que a torna cobiçada no mercado energético. Entretanto, a eficiência dos métodos de captação ainda provoca muita discussão, pois de forma contrária às fontes energéticas tradicionais, a energia solar depende diretamente de condições meteorológicas, da conversão dessa energia nas formas térmica ou elétrica e de modelos que otimizem a captação da luz solar.

Logo, uma das formas que possibilita o aprimoramento dessa captação é o direcionamento da estrutura captadora conforme o movimento aparente do Sol, segundo um referencial terrestre, o que é conhecido como rastreamento solar ou heliotropismo e pode ser observado inclusive em plantas.

Esse rastreamento pode ocorrer em um ou dois eixos e tem por objetivo, posicionar o captador solar de forma perpendicular aos raios solares incidentes, permitindo um melhor aproveitamento de captação. O presente estudo visa, portanto, apresentar tecnologias de rastreamento solar em um e dois eixos.

\section{MECANISMOS DE RASTREAMENTO SOLAR}


O Sol é uma esfera, feita de matéria gasosa intensamente quente, com um diâmetro de aproximadamente $1.39 .10^{9} \mathrm{~m}$ e, em média, situado a uma distância de $1,5.10^{11} \mathrm{~m}$ da Terra, sua temperatura efetiva, como um corpo negro, é de $5762 \mathrm{~K}$ adquirida através de constantes fusões nucleares (Duffie; Beckman, 2013). Ele gira sobre o seu eixo com uma frequência aproximada de uma vez a cada quatro semanas, no entanto, não gira com um corpo sólido, apresentando movimentos equatoriais mais rápidos que os polares. Observado à partir da Terra, o Sol se movimenta do sentido Leste para Oeste, com variações durante o ano, o que é conhecido como rastro, ou caminho do Sol. Logo, esse deslocamento, é a base para os mecanismos de rastreamento solar, conforme estudado por Mousazadeh et al. (2009).

\subsection{Posição e Movimento Solar}

A posição do Sol na esfera celeste foi convencionada em dois sistemas de coordenadas principais: a azimutal e a equatorial. O primeiro tem como referenciais dois ângulos, o azimute e a altitude, conforme ilustrado na Figura 1 e conceituado a seguir:

- Azimute: Medida que corresponde a uma distancia angular mensurada sobre o plano do horizonte, no intervalo de $0^{\circ}$ a $360^{\circ}$, a partir do Norte, no sentido horário.

- Altitude: Ângulo entre a projeção do Sol no plano de referencia e a posição do Sol. Também chamada de elevação solar.

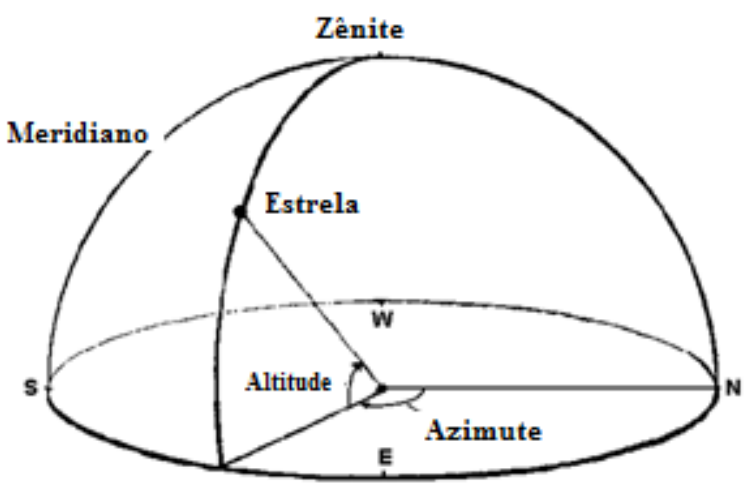

Figura 1- Relação entre ângulos de coordenadas azimutais. ${ }^{[1]}$

O segundo sistema, se baseia nos ângulos de ascensão reta e de declinação, conforme ilustrado na Figura 2. Esses ângulos são conceituados a seguir:

- Declinação: Ângulo entre o equador celeste e o astro em questão variando entre 0 e $90^{\circ}$. Quando se compara o sistema equatorial com o sistema de coordenadas geográficas, a declinação é análoga à latitude.

- Ascensão Reta: Ângulo entre o astro e a localização do Sol no Ponto Vernal (Equinócio de Março). Quando comparado ao sistema de coordenadas geográficas, a ascensão reta é análoga à altitude. 


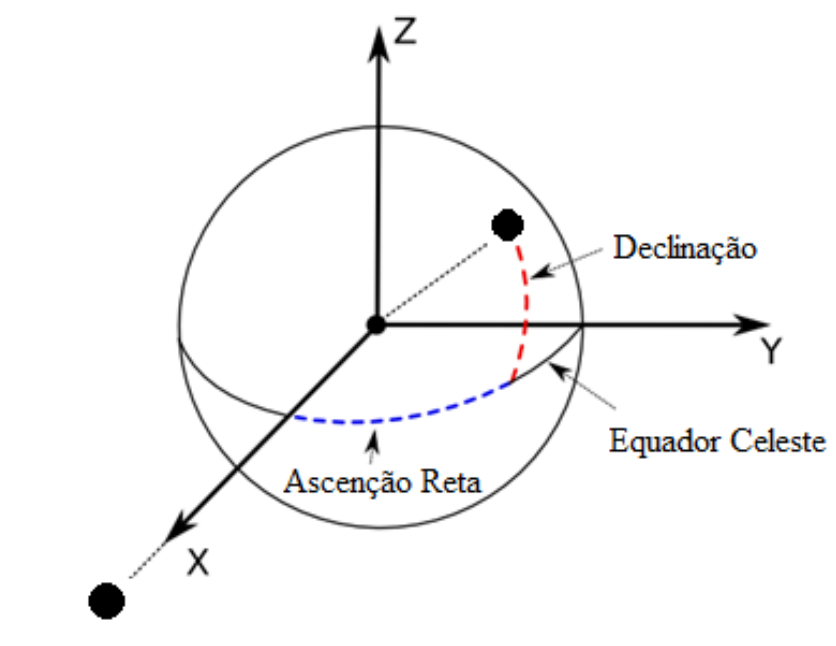

Ponto Vernal

Figura 2- Relação entre ângulos de coordenadas equatoriais. ${ }^{[1]}$

O Zênite é a linha imaginária que inicia sobre uma superfície seguindo perpendicularmente até a esfera celeste. O zênite solar ocorre quando o Sol está na sua posição mais alta em relação ao solo durante o dia, ou seja, ao meio-dia do tempo solar.

Para calcular a posição do Sol são utilizados como variáveis primárias o dia do ano, a latitude e o horário de interesse, Walraven (1978), Lamm (1981), Blanco-Muriel (2001), Grena (2008) e Chong e Wong (2009) são alguns dos autores que desenvolveram algoritmos para esses cálculos.

Acerca dos movimentos solares, a cada ano, a distância entre o Sol e a Terra muda o que é conhecido como solstícios e equinócios. Solstícios de verão apresentam maior janela solar que é caracterizada pela maior quantidade de horas de Sol por dia. Por outro lado, o solstício de inverno compreende um dia com menores quantidades de Sol, resultando em menor coleta dos raios solares. Os equinócios de primavera e outono são caracterizados pela duração igual dos dias e noites e são ilustrados na Figura 3.

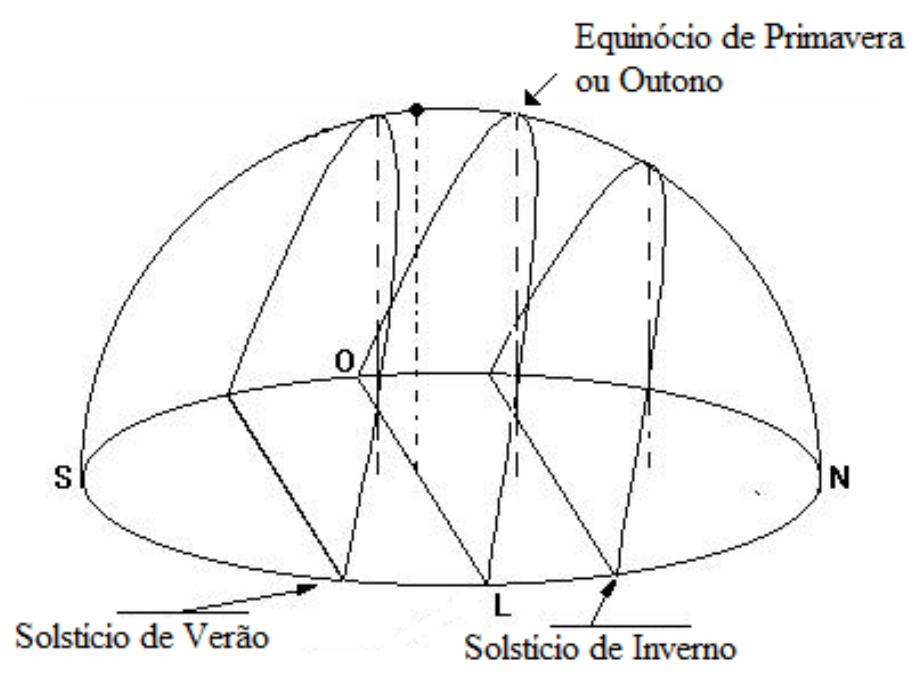

Figura 3 - Solstícios e Equinócios. ${ }^{[1]}$ 


\subsection{Heliotropismo Nas Plantas}

As plantas, em sua estrutura, são capazes de monitorar as movimentações do Sol através de um processo conhecido como heliotropismo. Esse processo é intimamente relacionado com o movimento foliar, que por sua vez, também sofre ação de diversos parâmetros, como a temperatura da folha, as condições de água, do solo e a quantidade de nitrogênio disponível.

Segundo Darwin (1881), existe dois tipos de heliotropismo, que são definidos a partir da estrutura foliar da planta. São eles:

- Diaheliotropismo: a lâmina foliar das plantas acompanha o Sol, mantendo-se perpendicular aos raios solares. Assim, a planta consegue aumentar sua captação de luz e o ganho de carbono.

- Paraheliotropismo: a lâmina foliar acompanha os movimentos do Sol mantendose paralela a incidência dos raios solares. Nesse caso, ocorre redução dos efeitos negativos relacionados ao transporte de água, foto inibição de raios UVB e redução da temperatura na folha, auxiliando na transpiração da planta.

Em um estudo realizado por Rakocevic et al. (2010), foram analisadas duas culturas de soja, "BR 16" e "Embrapa 48", as quais foram divididas em lotes irrigados e não irrigados. Após as etapas de tratamento do solo e plantação, foi possível notar a presença do heliotropismo atuando de diversas formas na plantação.

Para cada etapa do estudo, a elevação da planta e o azimute foram medidos a cada hora. Outro dado importante foi o equacionamento do ângulo entre os raios solares e a lamina foliar, Equação 1. Sendo, o ângulo entre os raios solares e a lamina foliar (i), o ângulo da folha com a horizontal $(\beta)$, o ângulo solar zenital $(\mathrm{z})$, o ângulo azimutal solar $\left(\mathrm{a}_{\mathrm{s}}\right)$ e o ângulo azimutal foliar $\left(\mathrm{a}_{1}\right)$.

$$
\cos i=\cos \beta \cdot \cos z+\operatorname{sen} \beta \cdot \operatorname{sen} z \cdot \cos \left(a_{s}-a_{1}\right)
$$

\subsection{Rastreamento Solar De Um Eixo}

Sistemas de rastreamento solar de um eixo são aqueles capazes de acompanhar o movimento solar em apenas uma faixa de abrangência, ou seja, variando apenas um ângulo, o que é ilustrado na Figura 4, tal qual o sistema polar ou tilt-roll.

Neste sistema, o modelo utiliza como referencial o eixo polar da Terra. Para uma melhor eficiência na coleta dos raios solares recomenda-se que o equipamento coletor esteja inclinado conforme a latitude local. $\mathrm{O}$ rastreamento solar ocorre em velocidade constante de $15 \%$ e pode ser montado tanto no sentido Leste-Oeste como no sentido Norte-Sul. 


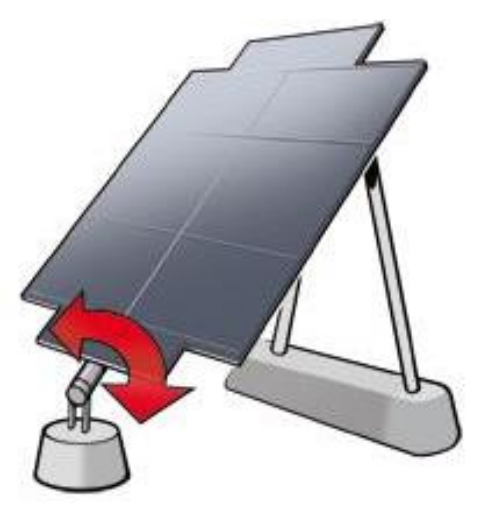

Figura 4 - Modelo de rastreamento solar de um eixo ${ }^{[2]}$.

No estudo de Oria e Sala (1988), foi feito um teste de módulos fotovoltaicos bifaciais aliados a um sistema polar de rastreamento. Essa mesma técnica de estudo também foi abordada no artigo de Faiman (1988), porém utilizando módulos fotovoltaicos triangulares. Acerca da estrutura de um modelo que acompanha esse sistema, Agee e Jimoh (2012) propõem diversas atualizações de protótipos que objetivem redução de custos através da mobilidade e movimentação do sistema integrado como um todo.

O rastreamento solar de um eixo tem como principal vantagem à facilidade de estruturar a movimentação do equipamento, uma vez que é realizado a uma velocidade constante, contudo, traz como empecilho uma menor eficiência de coleta, uma vez que o ângulo de inclinação fica fixo.

\subsection{Rastreamento Solar de Dois Eixos}

Sistemas de rastreamento solar de dois eixos são aqueles capazes de seguir o movimento solar nas duas faixas de abrangências, dessa forma, há um acompanhamento completo do movimento do Sol, nas duas variações angulares, conforme ilustra a Figura 5. A seguir, são apresentados os dois principais tipos de sistemas que utilizam esse rastreamento.

Sistema alto-azimutal: Sistema de rastreamento com variação angular azimutal e de altura, também chamada de inclinação, conforme ilustrado na Figura 5. Tem como principal vantagem à facilidade de montagem, porém o rastreamento exige mudança constante dos ângulos.

Esse sistema também apresenta alguns problemas, principalmente quanto a sua geometria. No estudo de Guo et al. (2011), foram destacados erros de inclinação em relação a linha vertical do azimute, a não-ortogonalidade dos eixos de rotação do heliostato e a falta de paralelismo entre as superfícies refletoras e o eixo de altitude. A redução desses, pode ser feita a partir de técnicas físicas e matemáticas.

Para ilustrar o efeito dos erros nos eixos, Lubitz (2011) mostra a variação de eficiência na coleta dos raios solares a partir de ajustes na inclinação dos eixos azimutais. Assim, a partir de comparações foi possível analisar o ganho de eficiência entre o uso de mecanismos de rastreamento e posições fixas de concentradores solares. Já Tanaka et al. (2009), utilizou esse 
modelo em um alambique verificando a possibilidade de obter ganhos de produtividade movendo a estrutura apenas uma vez por dia.

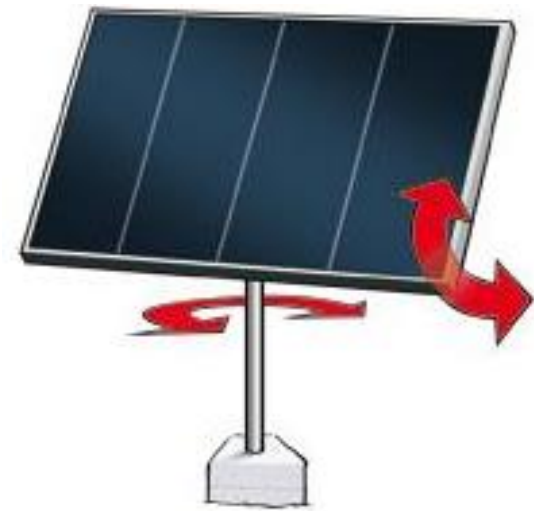

Figura 5- Modelo de rastreamento solar de dois eixos, alto-azimutal ${ }^{[2]}$.

\section{$\underline{\text { Sistema equatorial: }}$}

$\mathrm{O}$ rastreamento de dois eixos equatorial utiliza um sistema angular global e considera o eixo de ascensão reta (AR) e eixo de declinação (DEC), no qual o primeiro se refere ao alinhamento com o polo celeste, enquanto o segundo acompanha o movimento aparente do Sol. Tal modelo pode ser verificado no estudo de Mendoza et al. (2013), sobre aplicabilidade de células fotovoltaicas.

Os sistemas de rastreamento equatoriais são mais pesados e se leva mais tempo para ajustá-los, porém, uma vez alinhados com o polo, permitem acompanhar o Sol com movimentos em torno de um único eixo o que permite uma redução gradativa de movimentos motores e economiza energia nos movimentos de rastreamento, especialmente no torque atuante, alterando inclinações e ajustando os ângulos citados.

\section{RESULTADOS E DISCUSSÃO}

O rastreamento solar contribui para otimizar à forma de captação dos raios solares, permitindo, sobretudo um aproveitamento energético mais eficaz. No estudo de Rakocevic et al. (2010), os movimentos heliotrópicos foram feitos durante todo o período do dia, e pode-se notar que o ângulo entre os raios solares e a lamina foliar sofreu alterações a partir das mudanças de cultura e de irrigação. Tal comportamento, permitiu observar que o rastreamento solar serve como indicador para uma plantação bem sucedida.

No estudo de Oria e Sala (1988), o sistema de rastreamento de um eixo, apresentou não só um ganho de produtividade quanto comparado a um modelo estático, como redução dos custos envolvidos, alcançando níveis de produtividade 1,7 vezes maiores e uma redução de custos de $15 \%$.

Já no sistema de rastreamento de dois eixos, segundo Lubitz (2011), a utilização do rastreamento solar de um eixo manual, no sentido alto-azimutal, é capaz de garantir um ganho 
de eficiência de $29 \%$ na irradiação solar incidente, quando comparado a uma estrutura fixa afirmando, ainda, que essa inclinação depende do balanço entre o custo de trabalho e o ganho de energia após as modificações. Para tais ganhos de eficiência, deve-se estudar e mudar a inclinação de 2 a 12 vezes por ano.

Da mesma forma, Tanaka e Nakatake (2009), utilizaram-se da mudança diária dos ângulos alto-azimutal, e mediram a eficiência do alambique, obtendo uma média de $41 \%$ a mais na produtividade.

Quanto aos sistemas equatoriais, o custo dos protótipos de Agee e Jimoh (2012) foi reduzido em torno de $31 \%$, podendo permitir o uso de motores menores e possibilitando reduzir ainda mais o custo utilizando estruturas livres de sensores que controlam a movimentação do sistema polar. Os mecanismos de sistema equatorial conseguiram reduzir o valor do torque atuante a partir dos ângulos do mecanismo, resultando assim em uma redução da energia gasta no processo como um todo.

\section{CONCLUSÃO}

A necessidade de soluções sustentáveis faz da energia solar uma fonte fundamental de extração devido ao fato de ser limpa, renovável e praticamente inesgotável, contudo, ainda tem um baixo rendimento de conversão e captação. Para melhorar a eficiência de captação faz-se necessário acoplar ao equipamento coletor dos raios solares um sistema de rastreamento solar.

Este artigo apresentou mecanismos de rastreamento por um eixo que podem ser orientados nos sentidos Norte-Sul ou Leste-Oeste tendo como principal vantagem a simplicidade da movimentação feita em velocidade constante. O modelo de dois eixos foi dividido em duas categorias o alto-azimutal, de construção mais simples, porém com necessidade de interferência constante, e o equatorial que proporciona uma redução gradativa de movimentos motores, economizando energia nos movimentos de rastreamento, especialmente no torque atuante.

E por fim, foi visto que o rastreamento solar não é exclusividade das tecnologias mecânicas e elétricas sendo naturalmente realizado por plantas em um processo denominado heliotropismo. Dessa maneira, a utilização de um sistema de rastreamento solar é sugerido principalmente em meios que visam uma melhor produtividade tornando-se, em muitos casos, fundamental.

\section{AGRADECIMENTOS}

Os autores agradecem o apoio financeiro provido pela FAPEMIG (PCE-0020114: Participação Coletiva em Eventos Técnicos-Científicos)

\section{REFERÊNCIAS}

AGEE, J. T.; JIMOH, A. A. Flat controller design for hardware-cost reduction in polar-axis photovoltaic systems. Solar Energy, v.86, p.452-462, 2012. 
BLANCO-MURIEL, M.; ALARCON, P.D.C.; LOPEA, M.T., LARA, C.M. Computing the solar vector. Solar Energy, v.70, p.431-441, 2001.

CHONG, K. K.; WONG, C. W. General formula for on-axis sun-tracking system and its application in improving tracking accuracy of solar collector. Solar Energy, v.83, p.298-395, 2009.

DARWIN, C.; DARWIN, F. The Power of Movement in Plants, New York: D. Appleton \& Co, 1881 .

DUFFIE, J.A; BECKMAN, W.A. Solar Engineering of Thermal Process. New York: Wiley, 2013.

FAIMAN, D. Triangular photovoltaic modules for polar-axis trackers. Energy Conversion. Managemet, v.28, p.231-233, 1988.

GUO, M.; WANG, Z.; ZHANG, J.; SUN, F.; ZHANG, X. Accurate altitude-azimuth tracking angle formulas for a heliostat with mirror-pivot offset and other fixed geometrical errors. Solar Energy, v.85, p.1091-1100, 2011.

GRENA, R. An algorith for computation of the solar position. Solar Energy, vol.82, p. 462470, 2008.

LAMM L. O. A new analytic expression for the equation of time. Solar Energy, vol. 26, p. $465,1981$.

LUBITZ, W. D. Effect of manual tilt adjustments on incident irradiance on fixed and tracking solar panels. Applied Energy, v.88, p.1710-1719, 2011.

MOUSAZADEH, H.; KEYHANI, A.; JAVADI, A.; MOBLI, H.; ABRINIA, K.; SHARIFI, A. A review of principle and sun-tracking methods for maximizing solar systems output. Renewable and Sustainable Energy Reviews, V.13, P.1800-1818, 2009.

ORIA, J. P.; SALA, G. A good combination: Tracking of the Sun in polar axis and bifacial photovoltaic modules. Solar \& Wind Technology, v.5, p.629-636, 1988.

RAKOCEVIC, M.; NEUMAIER, N.; OLIVEIRA, G. M.; NEPOMUCENO, A. L.; FARIAS, J. R. B. Heliotropic responses of soybean cultivars at three phenological stages and under two water regimes. Pesq. agropec. bras., v.45, p.661-670, 2010.

TANAKA, H.; NAKATAKE, Y. One step azimuth tracking tilted-wick solar still with a vertical flat plate reflector. Desalination, v.235, p.1-8, 2009.

MENDOZA, J. M. G.; MONTÚFAR, C. P.; CAMPOS, J. A. F. Analytical synthesis for fourbar mechanisms used in a pseudo-equatorial solar tracker, Ingeniería e Investigación, v.33, p.55-60, 2013.

WALRAVEN, R. Calculating the position of the Sun. Solar Energy, v.20, 393-397, 1978.

[1] http://burro.cwru.edu/Academics/Astr306/Coords/coords.html <acesso em 05/04/2014>

[2] http://www.solarchoice.net.au/blog/solar-trackers/ <acesso em 05/04/2014> 